\title{
Quality of life in refractory generalized myasthenia gravis: A rapid review of the literature
}

\author{
Nathaly Garzón-Orjuela ${ }^{1, *}$, Laura van der Werf ${ }^{1}$, Laura Catalina Prieto-Pinto ${ }^{1}$, \\ Pieralessandro Lasalvia ${ }^{1,2}$, Camilo Castañeda-Cardona ${ }^{1}$, Diego Rosselli ${ }^{1,2}$ \\ 1. Department of Evidence-Based Medicine, NeuroEconomix, Bogotá, Colombia; \\ ${ }^{2}$ Clinical Epidemiology and Biostatistics Department, Medical School, Pontificia Universidad Javeriana, Bogotá, Colombia.
}

\begin{abstract}
Summary Generalized myasthenia gravis (GMG) is a neuromuscular transmission disorder that creates a fluctuating weakness of the voluntary muscles. This study is aimed at understanding the effect that refractory GMG has on the quality of life of patients who suffer from it, and the effect of eculizumab on it. A systematic literature search was conducted in MEDLINE (Ovid), EMBASE and the Cochrane Database of Systematic Reviews (Ovid). Eligibility criteria were verified via the title and summary and afterward through the full text. The risk of bias of the included randomized clinical trials was evaluated and the data were synthesized in a descriptive manner. Nine studies were identified that evaluated the quality of life of patients with GMG. Regarding the effect of eculizumab, two studies were identified. The quality of life in patients with GMG is lower compared to ocular myasthenia gravis (MG) and MG in remission, especially in the domains of physical function, physical role, bodily pain, vitality, and social function. Patients treated with eculizumab had a better perception of their quality of life compared to those who received placebo. GMG affects the quality of life more than other types of MG. This outcome is of great importance for the choice of therapeutic options in patients with refractory GMG. Eculizumab generates improvements in the perception of patients' quality of life compared to placebo, making it a relevant therapeutic option in the management of refractory GMG.
\end{abstract}

Keywords: Myasthenia gravis, refractory generalized myasthenia gravis, quality of life, generalized myasthenia gravis

\section{Introduction}

Myasthenia gravis (MG) is a disorder of neuromuscular transmission in which autoantibodies bind to acetylcholine receptors that are found in the postsynaptic membrane in the neuromuscular junction, making it difficult to transmit impulses to skeletal muscles and causing weakness $(1,2)$. Its prevalence varies between 15 to 179 cases per million and the mortality rate varies between 0.06 to 0.89 per million person-years (3).

This disease is characterized by fluctuating weakness of the voluntary muscles, which worsens with activity and as the day progresses $(2,4)$. It is classified as

\footnotetext{
*Address correspondence to:

Nathaly Garzón-Orjuela, Department of Evidence-Based Medicine, NeuroEconomix, Bogotá, Colombia.

E-mail: ngarzon@neuroeconomix.com
}

generalized or as ocular. In patients with ocular MG, the weakness affects only extraocular muscles and manifests itself with symptoms such as diplopia and palpebral ptosis (5). MG is considered generalized (GMG) when weakness involves the bulbar muscles, extremities or axial muscles. Weakness in the bulbar muscles causes difficulty in speaking, swallowing and chewing. In the muscles of the upper extremities, the weakness causes difficulty in raising the arms and performing daily tasks, such as combing, and when there is weakness in the muscles of the lower extremities, gait becomes unstable or irregular. Axial muscle involvement causes weakness in the back and neck, which can lead to painful spasms in these regions of the body. Weakness in these muscles is usually also accompanied by weakness in extraocular muscles $(5,6)$.

All these symptoms affect the quality of life of patients with GMG, and therefore the management 
of MG aims to restore muscle strength and decrease disease activity. There are mainly four types of therapy to achieve these goals: $i$ ) symptomatic therapy with acetylcholinesterase inhibitors; $i$ ) chronic immunosuppressive therapies; $i$ ii) rapid immunomodulatory therapies; and $i v$ ) Thymectomy $(7,8)$. However, between 10 and $30 \%$ of patients with MG do not respond adequately to conventional immunosuppressive therapy, so they require management with different types of biological therapy such as rituximab and eculizumab (7).

Therefore, this study was aimed at understanding the effect of refractory GMG on the quality of life of patients suffering from this disease (objective 1) and evaluating the effect of eculizumab on the quality of life of patients with refractory GMG (objective 2).

\section{Materials and Methods}

A systematic literature search was carried out through the design of two generic search strategies, delimited by key terms for each of the proposed objectives (see Table S1, http://www.irdrjournal.com/action/ getSupplementalData.php?ID=48). The following databases were searched: MEDLINE (OVID), EMBASE and the Cochrane Database of Systematic Reviews (OVID). Two independent researchers (LvdW and NG) performed an initial screening of the results by reviewing the title and summarizing the references obtained. Disagreements between the researchers were resolved by consensus. Subsequently, the eligibility criteria were verified (see Table S2, http://www.irdrjournal.com/ action/getSupplementalData.php?ID=48) using the full text of the preselected references.

The management and extraction of the data obtained from each included study were carried out using a form designed in Excel ${ }^{\circledR}$. This form included information on the main outcome of each study and the results of the quality of life outcome with its respective measurement scale. The risk of bias of the randomized clinical trials (RCTs) that were included was assessed, using the risk of bias tool designed by the Cochrane Collaboration. The data were synthesized descriptively by means of graphs or tables and grouped by the different scales of measurement of quality of life.

\section{Results}

After removing duplicates, a total of 193 references were identified. Following the review of the full text, 12 studies were included in the synthesis of evidence (see Figure 1). Table S3 (http://www.irdrjournal.com/ action/getSupplementalData.php?ID=48) shows the list of included and excluded studies for each objective, and Table S4 (http://www.irdrjournal.com/ action/getSupplementalData.php?ID=48) shows the characteristics of the included studies.

\subsection{Quality of life of patients with GMG}

The outcome of quality of life was identified in nine cross-sectional studies that focused on patients with GMG. No specific evidence was found for the subtype of refractory GMG. Five of the studies used the 36-Item Short Form Health Survey (SF-36) (9-13), three used the 15-item MG Quality of Life scale (MG-QOL15) (1416) and one used a specific Italian disease questionnaire (IMGQ) (see Table S4, http://www.irdrjournal.com/ action/getSupplementalData.php?ID=48) (17).

\subsubsection{Quality of life of patients with GMG measured with the SF-36 scale}

The SF-36 scale has a score of 0 to 100 that reflects the overall state of health and classifies it from worst to best state of health, in which lower scores represent
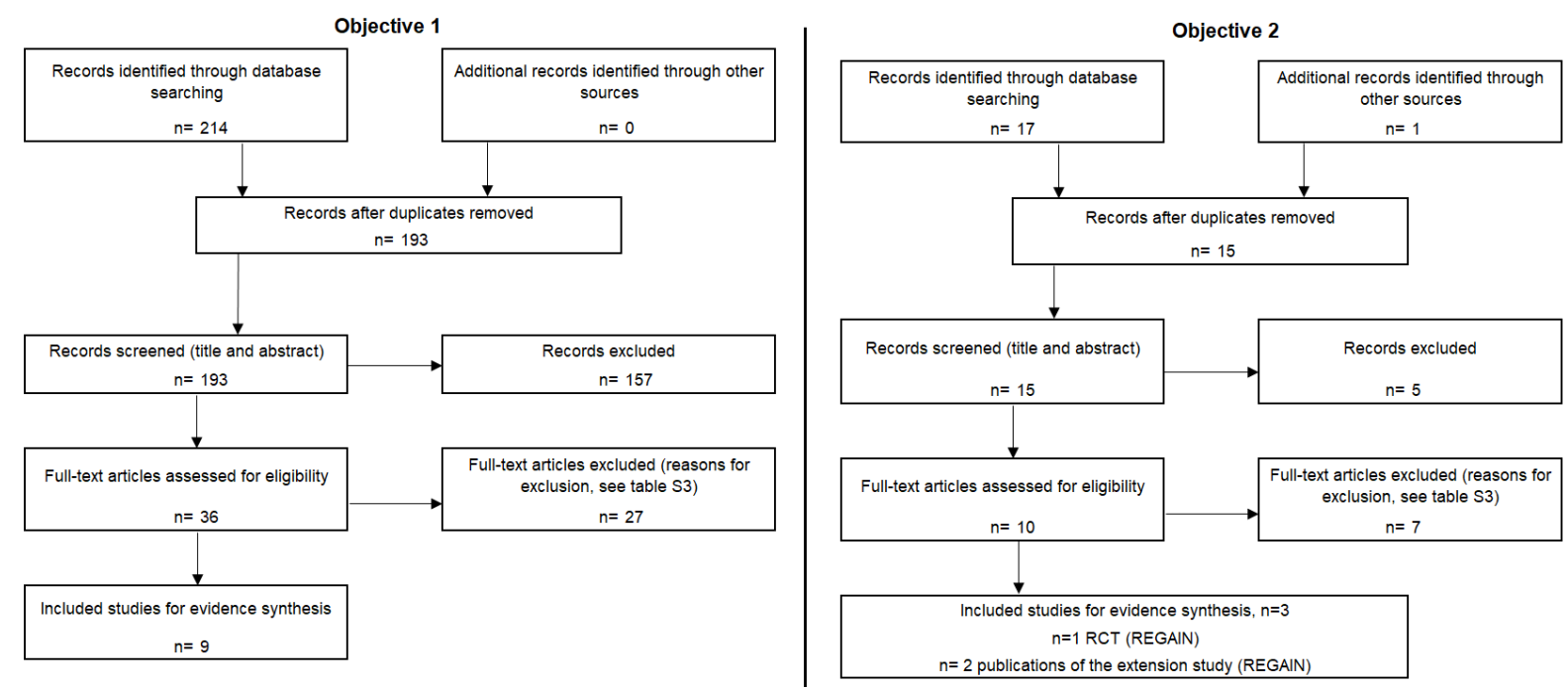

Figure 1. Flowchart search screening and selection of evidence. 
a worse state of health. It contains 36 questions organized in eight domains: physical function, physical role, bodily pain, general perception of health, social function, emotional role, mental health, and vitality; in some cases the first four domains are grouped into a general physical component and the remaining ones make up the mental component (10). In the study by Boldingh et al., patients with GMG had a lower score in the physical and mental component (mean values of 48 and 61 points, respectively) than patients with ocular $\mathrm{MG}$, in remission and residual ocular. When comparing these scores with the results of patients with ocular MG (patients who began with and remained with only ocular symptoms throughout the course of the disease), residual ocular (patients with residual ocular symptoms after having generalized disease) and in remission, the differences were statistically significant $(p<0.001)$ (see Figure 2) (10).

Likewise, in the study by Yang et al. (12), a comparison was made between two types of $\mathrm{MG}$ (GMG and ocular MG) by domain and quality of life component. Statistically significant differences were observed in the domain of physical function $(p<0.001)$, physical role $(p<0.001)$, bodily pain $(p=0.028)$, vitality $(p=0.018)$, social function $(p=0.003)$, and in the physical and mental health component in general $(p$ $<0.001$ and $p=0.022$, respectively) (12).

Two studies were also identified that compared the results $(11,12)$ by domain and overall quality of life score in patients with $\operatorname{GMG}(11,12)$, with a reference population (VR) of the study of Cohen et al. (18). In the first study, despite the small sample size $(n=20$ patients with GMG), statistically significant differences were identified when comparing the two populations in the domains of physical function (GMG: $57 \pm 22$ compared with VR: $84.5 \pm 22.9$ ), physical role (GMG: $47 \pm 40$ compared with VR:81.2 \pm 33.8) and general perception of health (GMG: $49 \pm 11$ compared with VR: $72.2 \pm$ 20.2) (11). In Figure 3, the results of the second study are observed, also with a small sample size $(n=29$ patients GMG), in which a lower score is also reported in the population with GMG in most domains, except for the mental health domain (12).

Finally, Basta et al. (9) performed a disease severity analysis according to the Myasthenia Gravis Foundation of America (MGFA) classification, where patients from Category II onward are considered to have GMG. It was identified that patients with severe MG have worse scores in all domains in the physical and mental health component in general, as well as in the overall quality of life score $(p=0.001)$.

\subsubsection{Quality of life of patients with GMG measured with the MG-QOL15 scale}

The MG-QOL scale consists of 15 items, each rated from 0 to 4 according to its frequency, with a maximum score of 60 . The higher the score, the worse the quality of life perceived by the patient (19). In the study by Hernández et al. (14) the quality of life of three types of MG was compared, finding that patients with GMG have a higher score on the MG-QOL scale $(19.3 \pm 10.5)$, that is, a worse perceived quality of life, compared to the reported scores in patients with $\mathrm{MG}$ in remission and ocular MG $(3.6 \pm 5.2$ and $8.8 \pm 7.4$, respectively).

The study by Hoffmann et al. in 2016 sought to assess the impact of fatigue, measured with the Chalder Fatigue Scale (CFQ). In general, it was shown that the quality of life scores were significantly higher in patients who displayed fatigue $(\mathrm{CFQ} \geq 4)$ in the three types of MG, patients with GMG having a worse perception of quality of life (mean of 22.7) compared with patients with MG in remission (mean of 8.6) and

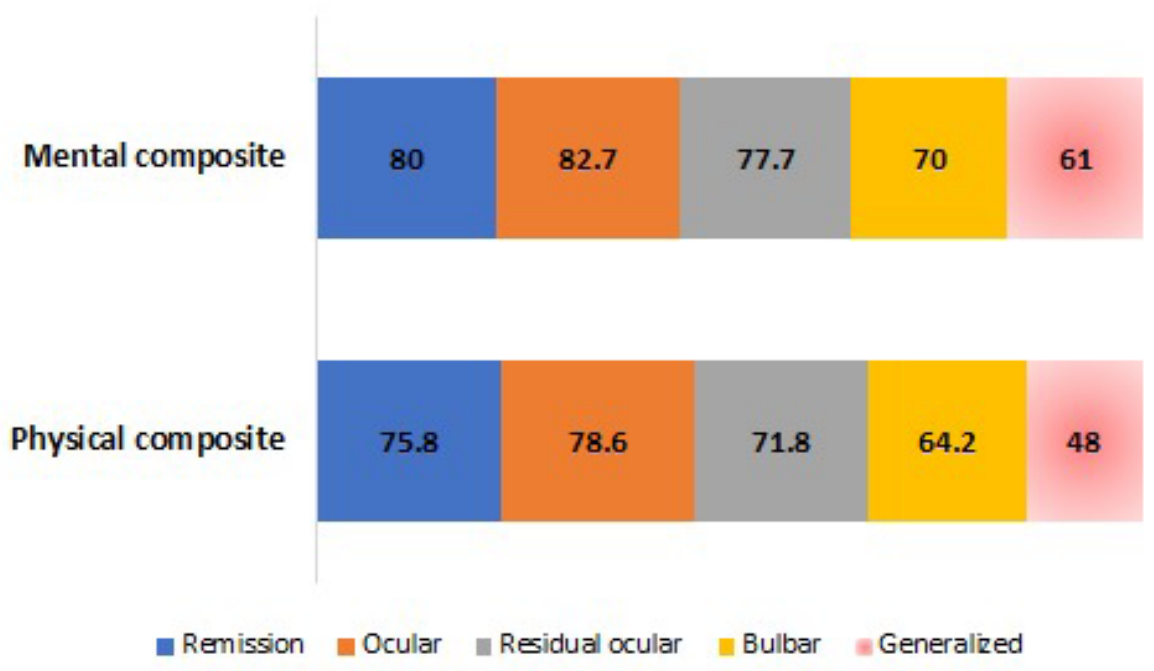

Figure 2. Score of the physical and mental component of the SF-36 scale by type of myasthenia gravis, in Boldingh et al. (10). Mean values. MG: Myasthenia gravis. Source: Adapted from Boldingh, et al. An up-date on health-related quality of life in myasthenia gravis - results from population-based cohorts. Health Qual Life Outcomes. 2015; 13:115. 


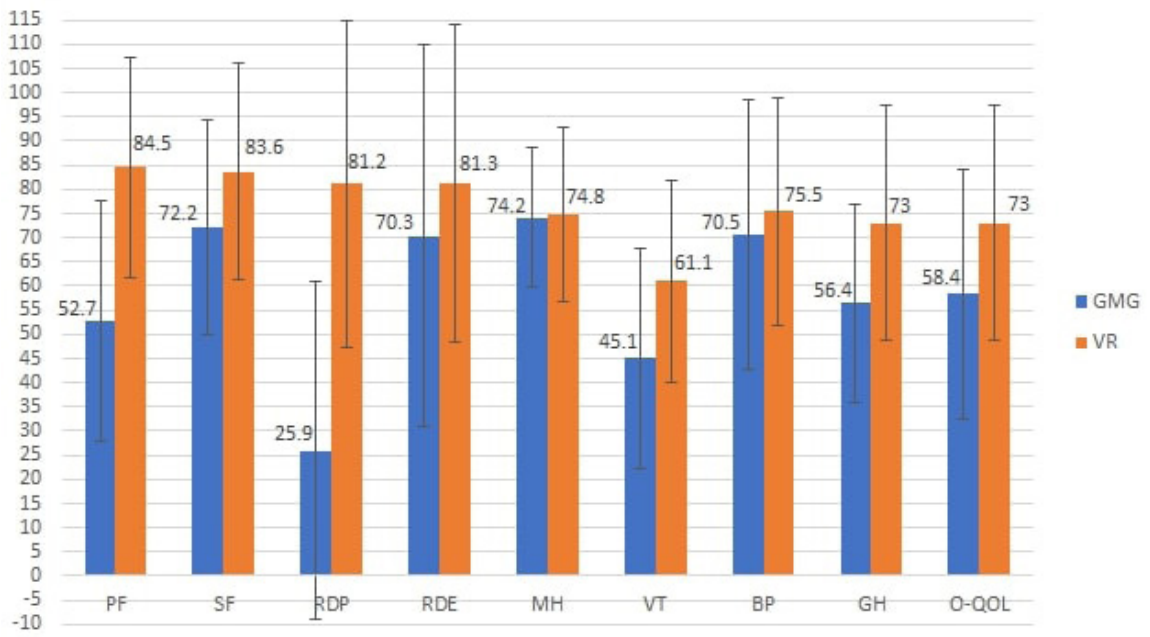

Figure 3. Score using the SF-36 scale by domain in the GMG population and reference population, in Paul et al. (12). Mean \pm standard deviation. GMG: Generalized myasthenia gravis, VR: Reference values, O-QOL: Overall quality of life related to health, PF: physical function, SF: social function, RDP: Role disruption-physical, RDE: Role disruption-emotional, MH: mental health, VT: vitality, BP: body pain, GH: general perception of health. Source: Adapted from Paul RH, et al. Quality of life and well-being of patients with myasthenia gravis. Muscle Nerve. 2001; 24:512-516.

ocular MG (mean of 19.3) (15).

Finally, in India Kumar et al. (16) performed an analysis of quality of life by the severity of the disease according to the MGFA classification, finding average values on the MG-QoL15 scale of 3.5, 9.4 and 15.9 in MG grades I, II and III/IV, respectively. A correlation was identified between the severity of the disease and the quality of life scores. Patients with grade I/II had a better perception of quality of life compared to the more severe degree of the disease (MGFA III/IV).

\subsubsection{Quality of life of GMG patients measured with the IMGQ questionnaire}

The IMGQ is a specific self-reported questionnaire for the disease, which assesses the perception of the quality of life. Its items are classed as good or bad (17). This questionnaire was used by Cioncoloni et al., whose study aimed to assess the changes in motor function that determine to a large extent the loss of a good quality of life in patients with MG. As a result of the evaluation of 41 patients (12 with ocular MG and 29 with GMG), 18 patients of the 29 with GMG reported their perception of quality of life as excellent or good, and 11 as poor, while none of those in the group with ocular MG reported a poor quality of life. Patients with a poor perception of quality of life showed a significantly lower overall IMGQ questionnaire score and a higher score on the scale of evaluation of symptoms and activities of daily living (the myasthenia gravisspecific activities of daily living scale (MG-ADL)) in comparison with patients who displayed an excellent or good perception of quality of life. In addition, in the analysis of variables associated with a poor quality of life, it was identified that the variables such as difficulty chewing solid foods and breathing while at rest (items of the MG-ADL scale) show a significant association with a poor perception of quality of life $(p<0.005)$ (OR: 6.57, $95 \%$ CI: 1.19 to 36 ; and OR:14, $95 \%$ CI: 1.11 to 176 , respectively) (17).

\subsection{Effect of eculizumab compared with placebo on the quality of life of patients with refractory $G M G$}

In the second objective, the outcome of quality of life was evaluated in a randomized, double-blind and multicenter phase III clinical trial (REGAIN) (20), with a low risk of bias (Table S5, http://www.irdrjournal. com/action/getSupplementalData.php? ID $=48)$, and its extension study (two publications) $(21,22)$.

This RCT evaluated the efficacy and safety of eculizumab compared with placebo in patients with refractory GMG from 17 centers in 17 countries in North America, Latin America, Europe, and Asia. Patients were randomly assigned to the eculizumab group or the placebo group until week 26. Administration for both eculizumab and placebo was performed as follows: 900 $\mathrm{mg}$ on the first day in week 2 and week 3; in week 4 they received a dosage of $1,200 \mathrm{mg}$, and after this the patients received $1,200 \mathrm{mg}$ of maintenance every two weeks (20). The following efficacy outcomes were evaluated: activities of daily living measured with the MG-ADL scale; severity or disability of the disease with the Quantitative Myasthenia Gravis (QMG) scale; clinical signs and symptoms with the Myasthenia Gravis Composite (MGC) scale; and quality of life (20).

The quality of life outcome was analyzed using a statistical model of worst-rank ANCOVA, in order to explain the potential effect of rescue medication in efficacy evaluations, as well as other negative outcomes (death, MG crisis, rescue use or treatment interruption). In this analysis (worst-rank) the patients were ranked 
from 1 (best outcome) to 125 (worst outcome), taking into account the time function until the event for the worst outcome category, and the change of the baseline until week 26 for the best outcome category (20). In addition to this first analysis, two additional analyses were performed:

i) Sensitivity analysis: patients with poor or negative outcomes were ranked according to the change from baseline until week 26 and not as a function of time until the event. For this approach, the worst-case ANCOVA analysis and a repeated measures analysis were used with the changes observed at each visit, adjusting them according to the use or non-use of immunosuppressive therapies as the covariate.

ii) Post-hoc sensitivity analysis: patients who left without experiencing death, myasthenic crisis, rescue therapy or worsening of the disease were included in the group of patients who did not experience negative outcomes. Patients who died (none in REGAIN), experienced myasthenic crises, or presented exacerbation of MG with or without the need for rescue therapy, were assigned to the worst ranks (death was classified as the worst outcome, followed by myasthenic crises, the requirement of rescue therapy, and interruption due to disease progression).

The outcome of quality of life was evaluated with the MG-QoL15 scale; the average baseline score for the intervention group was $33.6 \pm 12.2$ and that for the placebo group was $30.7 \pm 12.7$ (20). In Table 1 , the results of the ANCOVA analysis and repeated measures (worst-rank) of the change of the baseline until week 26 are observed. In the analysis of repeated measures without immunosuppressive therapies as a covariate, patients who received eculizumab showed a better score in week 4 compared to that of the baseline, and a greater effect at week 12 and 26. The average change between baseline until week 26 was greater for the eculizumab group in the analysis of repeated measures with and no immunosuppressive therapies as covariate (20) (Table 1).

Between the REGAIN study and its extension, a blind induction phase of 4 weeks was performed in order to preserve the masking nature of the REGAIN study. During this phase, the researchers, patients, and personnel of the study remained blind to all treatment assignments. Patients who had been assigned to eculizumab in REGAIN received eculizumab $1200 \mathrm{mg}$ (4 vials) on the first day and in week 2, and placebo (4 vials) in weeks 1 and 3. Patients who had been assigned to placebo in REGAIN received eculizumab (900 mg, 3 vials) + placebo (one vial) every week. After this phase (week 4), all patients received eculizumab with an openlabel (1,200 mg) every 2 weeks (22).

Two baselines were taken into account in the extension study for data analysis: the first day of the REGAIN study, and the last evaluation available before the first infusion of eculizumab in the extension study. These analyses were based on repeated measurement models. With regards to the change measured from the first baseline (first day of the REGAIN study), the result of quality of life on the MG-QoL15 scale reported by patients who received eculizumab during the 26 weeks of follow-up was identified as being maintained during the extension (cut-off point for analysis at three years) (22).

In the repeated measures analysis, performed to evaluate the change from the baseline of the extension study until week 130, no significant differences were found in the eculizumab/eculizumab group (average change of -1.2 from the start to the week 130 of the extension study, $p=0.4756$ ), while patients who received placebo during the REGAIN study experienced a rapid improvement in quality of life when they started treatment with eculizumab, displaying an average change of -5.4 from the baseline to the 130th week of the extension study $(p<0.0001)(22)$.

Table 1. Quality of life results (MG-QoL15) of the REGAIN study

\begin{tabular}{|c|c|c|c|c|}
\hline Items & $\begin{array}{l}\text { Eculizumab } \\
\qquad(n=62)\end{array}$ & $\begin{array}{l}\text { Placebo } \\
(n=63)\end{array}$ & Difference $(95 \% \mathrm{CI})$ & $p$ value \\
\hline Worst-rank ANCOVA analysis score * & $55.5(4.6)$ & $69.7(4.5)$ & $-14.3(-27$ to -1.6$)$ & 0.0281 \\
\hline Sensitivity analysis with the worst-rank ANCOVA analysis score * & $55.6(4.6)$ & $69.5(4.5)$ & $-13.9(-26.6$ to -1.2$)$ & 0.0328 \\
\hline Sensitivity analysis with the change in scale score - ANCOVA ** & $-11.3(1.5)$ & $-6(1.5)$ & $-5.2(-9.4$ to -1.0$)$ & 0.0152 \\
\hline $\begin{array}{l}\text { Sensitivity analysis with a model of repeated measures with immunosuppressive } \\
\text { therapy as a covariate } * *\end{array}$ & $-13.8(1.6)$ & $-6.7(1.6)$ & $-7.1(-11.3$ to 3$)$ & 0.0009 \\
\hline $\begin{array}{l}\text { Sensitivity analysis with a model of repeated measures without the use of } \\
\text { immunosuppressive therapy as a covariate } * *\end{array}$ & $-12.6(\mathrm{NR})$ & $-5.4(\mathrm{NR})$ & $-7.2(\mathrm{NR})$ & 0.0010 \\
\hline Post-hoc sensitivity analysis of worst-rank ANCOVA* & $54.6(4.5)$ & $70.6(4.5)$ & $-16(-28.6$ to -3.4$)$ & 0.0134 \\
\hline
\end{tabular}

NR: Data not reported in the study. ${ }^{*}$, Change in the score from the baseline until week 26 - worst-rank ANCOVA presented as the mean of the range of least squares. ${ }^{* *}$, Change in score from baseline until week 26 or last observation of the MG-QoL15 scale score given as the mean of least squares. Source: Howard JF, et al. Safety and efficacy of eculizumab in anti-acetylcholine receptor antibody-positive refractory generalised myasthenia gravis (REGAIN): a phase 3, randomised, double-blind, placebo-controlled, multicentre study. Lancet Neurol. 2017; 16:976-986. 
Table 2. Results of the outcome of fatigue (Neuro-QOL) of the patients of the REGAIN study and its extension

\begin{tabular}{|c|c|c|}
\hline Items & Intervention $(95 \% \mathrm{CI})$ & Control $(95 \% \mathrm{CI})$ \\
\hline Mean difference between baseline and week 26 of the REGAIN study & $\begin{array}{l}-16.3 \\
(-20.8 \text { to }-11.8)\end{array}$ & $\begin{array}{c}-7.7 \\
(-12.1 \text { to }-3.3)^{*}\end{array}$ \\
\hline $\begin{array}{l}\text { Mean difference between the baseline of the REGAIN study and week } 4 \text { of the } \\
\text { extension study }\end{array}$ & $\begin{array}{c}-17.8 \\
(-22.5 \text { to }-13)\end{array}$ & $\begin{array}{c}-17.4 \\
(-22 \text { to }-12.9)\end{array}$ \\
\hline $\begin{array}{l}\text { Mean difference between the baseline of the REGAIN study and week } 54 \text { of the } \\
\text { extension study }\end{array}$ & $\begin{array}{l}-17.5 \\
(-22.5 \text { to }-12.5)\end{array}$ & $\begin{array}{l}-15.7 \\
(-20.5 \text { to }-10.9)\end{array}$ \\
\hline
\end{tabular}

$* p=0.0081$. Source: Andersen H, Mantegazza R, Wang JJ, O'Brien F, Patra K, Howard JF Jr; REGAIN Study Group. Eculizumab improves fatigue in refractory generalized myasthenia gravis. Qual Life Res. 2019; 28:2247-2254.

Andersen et al. (21) performed an analysis with the data from the REGAIN study and its extension by correlating the fatigue data, measured by the Quality of Life in Neurological Disorders (Neuro-QOL) subscale (higher scores indicate greater fatigue) and quality of life scores (MG-QOL15). In Table 2, the fatigue results of the patients of the REGAIN study and their extension are observed. They also identified a strong, statistically significant correlation between fatigue scores and quality of life scores when the change from baseline to week 26 of the REGAIN study was measured. This correlation was evident in both the eculizumab group and the placebo group (21).

\section{Discussion}

This study sought to identify the effect or impact of refractory GMG on the quality of life of patients. As a result of the search, 9 cross-sectional studies were identified that assessed the quality of life of patients with GMG; however, no specific evidence was identified for the subtype of refractory GMG. In most of the included studies, comparisons are made between different types of MG, identifying a greater impact on the quality of life in GMG versus ocular MG and MG in remission $(10,13,14)$. The differences are especially found in the domains of physical function, physical role, bodily pain, vitality and social function (13). The connection of the domains of quality of life may be related to that found by Oosterhuis $\mathrm{H}$, in his study of the natural course of MG in 1989, in which he noted that when GMG is associated with predominant bulbar muscle weakness, it results in severe restrictions in the activities of daily life and, when severe, it can cause myasthenic crises or require respiratory assistance (23). Likewise, in the study of Basta et al. (9) a relationship between the severity of the disease and worse scores on the quality of life scales was described, both in the physical component and in the mental health component. Similar results were identified in the study of Kumar et al. (16) where it was observed that patients with mild grades of the disease have a better quality of life compared to those with more severe grades.

Although no studies were found aimed at identifying predictive factors in the perception of quality of life of patients with GMG, it has been found in populations with MG that the change in life, that is, the change in occupation or work due to physical difficulty, and depression are significant factors in predicting the low quality of life of these patients. Since this disease is a chronic condition and there is no curable alternative, patients are more vulnerable to suffer, in addition to motor disorders, depression and low self-esteem that affect the mental health component, compromising the overall quality of life (24). As a result, it is essential to keep these factors in mind with any subtype of the disease, in order to propose and implement strategies that help positively influence the quality of life of these patients and their caregivers.

On the other hand, some authors have observed that the measurement of the quality of life in patients with MG at the time of evaluating and choosing a therapeutic option is of great help, because this measurement evaluates the impact of therapy at intermediate levels of clinical improvement and morbidity, in addition to supplementing the information provided by the analysis of clinical outcomes $(25,26)$. Likewise, patients with MG are often treated successfully, using therapies such as pyridostigmine, therapeutic plasma exchange (plasmapheresis or PLEX/PEX), corticosteroids, azathioprine, cyclosporine, and thymectomy, thus improving their life expectancy (27). However, a group of patients continues to perceive a reduction in their quality of life, especially patients with generalized symptoms or active disease (10), particularly patients with the disease that is resistant to treatment, who require more aggressive therapeutic options to prevent life-threatening myasthenic crises (28).

There are few therapeutic options for this last subtype of the disease (refractory GMG) becoming an unfulfilled requirement (29). Therapies such as rituximab have shown some benefits in small studies and individual cases of refractory MG, and other therapies are under investigation, such as rozanolixizumab and abatacept (30). Currently, eculizumab is the only alternative with phase III studies approved for these symptoms. Because of this, the second research question in the present study was in 
order to understand the effect of eculizumab on the quality of life in patients with refractory GMG. As a result of the search, three publications were identified: an RCT, the REGAIN study, and its extension.

Overall, it was shown that patients treated with eculizumab had a better perception of their quality of life when compared to the control group (placebo) and this effect was maintained during the three-year extension phase $(20,22)$. In addition, a statisticallysignificant rapid improvement in the quality of life score was demonstrated in the group of patients who received placebo during the REGAIN study and started treatment with eculizumab at the beginning of the extension phase (22). Andersen et al. (21) found a correlation between fatigue scores and quality of life, that is, greater fatigue resulted in lower quality of life, and the REGAIN study also identified that fatigue reported by patients improved more in the group treated with eculizumab than in the control group (22).

As a result eculizumab has become a novel therapy for this group of patients and in recent years has received approval by regulatory bodies in some countries such as the United States for the management of GMG $(29,31)$, in the European Union for refractory GMG $(29,32)$ and in Japan for the treatment of GMG with symptoms difficult to control with high doses of immune therapy (IgG or PLEX) $(29,33)$, in addition to its inclusion in clinical guidelines as a therapeutic option for the management of patients with severe and refractory GMG $(29,34)$.

\section{Conclusion}

The effects of health-related quality of life are higher in patients with GMG compared to other types of $\mathrm{MG}$, especially in the domains of physical function, pain, vitality, and social function. The measurement of this outcome in patients with MG, in the evaluation and choice of therapeutic options, is of great importance, especially in patients with refractory GMG where these options are scarce. In recent years, eculizumab has become a novel therapy for patients with refractory GMG because it generates improvements in the perception of patients' quality of life compared to placebo.

\section{Acknowledgements}

The work was funded by Alexion Pharma Colombia.

\section{References}

1. Gilhus NE. Myasthenia and the neuromuscular junction. Curr Opin Neurol. 2012; 25:523-529.

2. Gilhus NE. Myasthenia Gravis. N Engl J Med. 2016; 375:2570-2581.

3. Carr AS, Cardwell CR, McCarron PO, McConville J. A systematic review of population based epidemiological studies in Myasthenia Gravis. BMC Neurol. 2010; 10:46.

4. Silvestri NJ, Wolfe GI. Myasthenia gravis. Semin Neurol. 2012; 32:215-226.

5. Luchanok V, Kaminski HJ. Natural History of Myasthenia Gravis. Engel A, ed. 2012; pp. 90-107.

6. Keesey JC. Clinical evaluation and management of myasthenia gravis. Muscle Nerve. 2004; 29:484-505.

7. Gilhus NE, Tzartos S, Evoli A, Palace J, Burns TM, Verschuuren JJGM. Myasthenia gravis. Nat Rev Dis Primers. 2019; 5:30.

8. Ropper AH, Samuels MA, Klein JP. Chapter 49. Myasthenia Gravis and Related Disorders of the Neuromuscular Junction. The McGraw-Hill Companies, New York, 2014.

9. Basta IZ, Pekmezović TD, Perić SZ, Kisić-Tepavčević DB, Rakočević-Stojanović VM, Stević ZD, Lavrnic DV. Assessment of health-related quality of life in patients with myasthenia gravis in Belgrade (Serbia). Neurol Sci. 2012; 33:1375-1381.

10. Boldingh MI, Dekker L, Maniaol AH, Brunborg C, Lipka AF, Niks EH, Verschuuren JJ, Tallaksen CM. An update on health-related quality of life in myasthenia gravis -results from population based cohorts. Health Qual Life Outcomes. 2015; 13:115.

11. Fregonezi GA, Regiane-Resqueti V, Pradas J, Vigil L, Casan P. The relationship between lung function and health-related quality of life in patients with generalized myasthenia gravis. Arch Bronconeumol. 2006; 42:218224. (in Spanish)

12. Paul RH, Nash JM, Cohen RA, Gilchrist JM, Goldstein JM. Quality of life and well-being of patients with myasthenia gravis. Muscle Nerve. 2001; 24:512-516.

13. Yang Y, Zhang M, Guo J, Ma S, Fan L, Wang X, Li C, Guo P, Wang J, Li H, Li Z. Quality of life in 188 patients with myasthenia gravis in China. Int J Neurosci. 2016; 126:455-462.

14. Hernández E, Aguirre MEE, Blanco TA, Pascual IJ. Myasthenia gravis: Sleep quality, quality of life, and disease severity. Muscle Nerve. 2012; 46:174-180.

15. Hoffmann S, Ramm J, Grittner U, Kohler S, Siedler J, Meisel A. Fatigue in myasthenia gravis: Risk factors and impact on quality of life. Brain Behav. 2016; 6:e00538.

16. Kumar R, Nagappa M, Sinha S, Taly A, Rao S. MGQoL-15 scores in treated myasthenia gravis: Experience from a university hospital in India. Neurol India. 2016; 64:405-410

17. Cioncoloni D, Casali S, Ginanneschi F, Carone M, Veronica B, Rossi A, Giannini F. Major motor-functional determinants associated with poor self-reported healthrelated quality of life in myasthenia gravis patients. Neurol Sci. 2016; 37:717-723.

18. Cohen RA, Moser DJ, Clark MM, Aloia MS, Cargill BR, Stefanik S, Albrecht A, Tilkemeier P, Forman DE. Neurocognitive functioning and improvement in quality of life following participation in cardiac rehabilitation. Am J Cardiol. 1999; 83:1374-1378.

19. Taşcilar NF, Saraçli Ö, Kurçer MA, Ankarali H, Emre U. Reliability and validity of the Turkish version of myasthenia gravis-quality of life questionnaire-15 item. Turk J Med Sci. 2016; 46:1107-1113.

20. Howard JF Jr, Utsugisawa K, Benatar M, Murai H, Barohn RJ, Illa I, Jacob S, Vissing J, Burns TM, Kissel JT, Muppidi S, Nowak RJ, O'Brien F, Wang JJ, Mantegazza R; REGAIN Study Group. Safety and 
efficacy of eculizumab in anti-acetylcholine receptor antibody-positive refractory generalised myasthenia gravis (REGAIN): a phase 3, randomised, double-blind, placebo-controlled, multicentre study. Lancet Neurol. 2017; 16:976-986.

21. Andersen H, Mantegazza R, Wang JJ, O'Brien F, Patra K, Howard JF Jr; REGAIN Study Group. Eculizumab improves fatigue in refractory generalized myasthenia gravis. Qual Life Res. 2019; 28:2247-2254.

22. Muppidi S, Utsugisawa $\mathrm{K}$, Benatar $\mathrm{M}$, et al. Longterm safety and efficacy of eculizumab in generalized myasthenia gravis. Muscle Nerve. 2019; 60:14-24.

23. Oosterhuis HJ. The natural course of myasthenia gravis: A long term follow up study. J Neurol Neurosurg Psychiatry. 1989; 52:1121-1127.

24. Jeong A, Min JH, Kang YK, Kim J, Choi M, Seok JM, Kim BJ. Factors associated with quality of life of people with myasthenia gravis. PLoS One. 2018; 13:e206754.

25. Jaretzki A 3rd, Barohn RJ, Ernstoff RM, Kaminski HJ, Keesey JC, Penn AS, Sanders DB. Myasthenia gravis: recommendations for clinical research standards. Task Force of the Medical Scientific Advisory Board of the Myasthenia Gravis Foundation of America. Neurology. 2000; 55:16-23.

26. Jones SM, Gwathmey K, Burns TM. Quality of life measures for myasthenia gravis and evaluation of nonmotor symptoms. Clin Exp Neuroimmunol. 2015; 6:32-39.

27. Sieb JP. Myasthenia gravis: an update for the clinician. Clin Exp Immunol. 2014; 175:408-418.

28. Silvestri NJ, Wolfe GI. Treatment-Refractory Myasthenia
Gravis. J Clin Neuromuscul Dis. 2014; 15:167-178

29. Dhillon S. Eculizumab: A review in generalized myasthenia gravis. Drugs. 2018; 78:367-376.

30. Schneider-Gold C, Hagenacker T, Melzer N, Ruck T. Understanding the burden of refractory myasthenia gravis. Ther Adv Neurol Disord. 2019; 12:1756286419832242.

31. Alexion Pharmaceuticals I. Soliris (eculizumab): US prescribing information. 2017. https://www.accessdata. fda.gov/drugsatfda_docs/label/2011/125166s172lbl.pdf (accessed July 10, 2019)

32. Alexion Europe SAS. Soliris (eculizumab): summary of product characteristics. 2017. https://www.ema.europa.eu/ en/documents/product-information/soliris-epar-productinformation_en.pdf (accessed July 10, 2019)

33. Alexion Pharmaceuticals, Inc. Soliris ${ }^{\circledR}$ (Eculizumab) Receives Marketing Authorization In Japan For The Treatment Of Patients With Generalized Myasthenia Gravis (GMG). 2007. https://news.alexion.com/pressrelease/product-news/soliris-eculizumab-receivesmarketing-authorization-japan-treatment-patie (accessed July 10, 2019)

34. Melzer N, Ruck T, Fuhr P, Gold R, Hohlfeld R, Marx A, Melms A, Tackenberg B, Schalke B, Schneider-Gold C, Zimprich F, Meuth SG, Wiendl H. Clinical features, pathogenesis, and treatment of myasthenia gravis: A supplement to the Guidelines of the German Neurological Society. J Neurol. 2016; 263:1473-1494.

(Received October 26, 2019; Revised November 20, 2019; Accepted November 25, 2019) 